\title{
Retrospective Evaluation of Childhood Cutaneous Mastocytosis Cases
}

\author{
(1) Burcu Akıncı, (1) Hamiyet Hekimci Özdemir, (1) Nihal Özdemir Karadaş, (•) Akkız Şahin Yaşar, \\ (1) Zuhal Önder Siviş, (1) Yeşim Aydınok, (1) Kaan Kavaklı, (1) Can Balkan, (1) Deniz Yılmaz Karapınar \\ Ege University Hospital, Clinic of Pediatric Hematology and Oncology, İzmir, Turkey
}

\begin{abstract}
Aim: Mastocytosis is a rare disease characterized by clonal mast cell proliferation in one or more organs. It can lead to different clinical manifestations and has no definitive treatment. In this study, we aimed to evaluate the clinical and laboratory characteristics of our patients diagnosed with mastocytosis in our clinic.

Materials and Methods: Thirteen patients that were followed up with the diagnosis of mastocytosis at the Pediatric Hematology Clinic, in the Ege University Faculty of Medicine between November 1999 and April 2016 were retrospectively analyzed.

Results: Seven of patients were female (53.8\%) and six were male (42.6\%). The mean age at diagnosis was 20 (3-68) months. At the time of diagnosis, complete blood count and peripheral smear were found to be compatible with the anemia of iron deficiency in three patients. Other parameters were normal. Mean tryptase level was detected as 5.9 (3.6-16.6) ng/mL, and only one tryptase level was found as slightly increased. The median level of total IgE was 91.1 (4.47-362) IU/mL. Mast cell proliferation was not detected in bone marrow aspiration and biopsy material of any patients. All of the cases were evaluated as cutaneous type mastocytosis.

Conclusion: The possibility of mastocytosis in systemic form in childhood is very rare and bone marrow examination may be necessary in selected cases.
\end{abstract}

Keywords: Mastocytosis, cutaneous, tryptase, child

\section{Introduction}

Mastocytosis is a very rare disease that can lead to different clinical presentations depending on the tissues where clonal mast cells accumulate. The clinical spectrum of the disease may vary from relatively benign forms with isolated skin involvement to highly aggressive severe forms in which widespread systemic involvement results in poor prognosis. Over the years, significant progress has been made in the management of mastocytosis seen in the adulthood; however, childhood mastocytosis has remained a rarely researched topic due to the rareness of interventional procedures (1-4).

Mast cells, which originate from CD34 + hematopoietic cells, are considered as an important component of the immune system. They are present in all vascularized tissues, primarily the skin and mucous membranes. The growth, differentiation and proliferation of mast cells are controlled by c-kit (CD117), a tyrosine kinase receptor, and its ligand, stem cell factor. These factors are also involved in the pathogenesis of mastocytosis $(5,6)$. 
According to the criteria published by the World Health Organization in 2016, adult type mastocytosis was divided into three groups as cutaneous mastocytosis (CM), systemic mastocytosis (SM) and mast cell sarcoma. Cutaneous type mastocytosis is localized to the skin and is examined under three subgroups which are maculopapular mastocytosis (urticaria pigmentosa), diffuse CM and soliter mastocytoma. SM is characterized by mast cell accumulation in internal organs such as bone marrow, spleen, lymph nodes, and the gastrointestinal tract. SM is divided into subtypes such as indolent $S M$, smoldering $S M$, SM with an associated hematologic neoplasm, aggressive SM and mast cell leukemia $(7,8)$.

SM is defined by major and minor criteria. Major criteria is the multifocal dense infiltrate of mast cells in the bone marrow or in other extracutaneous organ(s) (>15 mast cells per aggregate). Minor SM criteria are: (a) $25 \%$ of mast cells show an abnormal morphology in bone marrow smears, or are spindle-shaped cells in biopsies of extracutaneous organ(s); (b) a KIT mutation at codon 816 in extracutaneous organ(s) or bone marrow; (c) Expression of CD2 and/or CD25 in bone marrow or extracutaneous organ(s); (d) serum tryptase $>20 \mathrm{ng} \mathrm{mL}-1$. If at least one major and one minor or at least three minor criteria are fulfilled, the diagnosis of SM is given $(1,3,8)$. Diagnostic criteria of SM are presented in Table I. Although mastocytosis is rarely seen in systemic forms in childhood CM localized to the skin is much more frequent), it has been reported that some of these cases do not enter into remission after adolescence and the severity of the disease may increase (1-4). In this study, we aimed to evaluate the clinical and laboratory findings of cases with a mastocytosis diagnosis in our clinic.

\section{Materials and Methods}

Thirteen patients diagnosed with mastocytosis in the Pediatric Hematology Clinic of Ege University Medical

Table I. Diagnostic criteria for systemic mastocytosis

Major

1. Multifocal dense infiltrates of mast cells (tryptase positive) in bone marrow and/or other extracutaneous tissues (aggregates of more than 15 mast cells).

\section{Minor}

1. More than $25 \%$ of the mast cells in bone marrow smears or tissue biopsy sections are spindle shaped or display atypical morphology.

2. Detection of a c-kit point mutation in codon 816 in blood, bone marrow, or other lesional tissue.

3. Evidence of CD2 and/or CD25 on mast cells in bone marrow,

blood, or extracutaneous tissue.

4. Serum tryptase more than $20 \mathrm{ng} / \mathrm{mL}$
Faculty from November 1999 to April 2016 were included in this study. Ethical approval was obtained (Acıbadem University ATADEK, 2019-19/11). Patients were accepted into the study after having given written informed consent from parents. All diagnoses were confirmed via histopathological investigation of skin biopsy samples. Evaluations were performed by retrospective evaluation of patient files. The patients' clinical findings, complete blood count results, biochemical parameters, tryptase levels, total Ig E levels, and the results of abdominal ultrasonography and bone marrow examinations were recorded.

\section{Statical Analysis}

All demographic and clinical wariables were summarized using count and percentage $n(\%)$ for categorical variables and means plus or minus standard deviations for continuous variables.

\section{Results}

Our patient group was comprised of 7 girls and 6 boys. The mean age at diagnosis was 20 (3-68) months. Patients' medical records showed that all applications to the department were due to skin rash. None of the patients had a family history of this disease. There were no pathological findings except mastocytosis-specific rashes on physical examination of the patients. The rashes were mostly located on the trunk. They were also seen in extremities and face. At the time of diagnosis, complete blood count and peripheral smear were found to be compatible with the iron deficiency anemia in three patients. Other parameters were normal. Mean absolute eosinophil count was 421.4/ $\mu \mathrm{L}(70-980 / \mu \mathrm{L})$. Liver and kidney function tests and other biochemical values were within their respective reference ranges. Abdominal ultrasonography was performed in 12 patients. In one patient, liver calcification was detected incidentally and the patient was diagnosed with hydatid cyst afterwards. The other patients had normal ultrasonography findings. Total tryptase levels had been measured in all but one of our patients. Mean tryptase level was 5.9 (3.6-16.6) $\mathrm{ng} / \mathrm{mL}$. In only one of our patients, the level of tryptase was found to be slightly increased $(16.6 \mathrm{ng} / \mathrm{mL})$; however, on subsequent measurements ( 3 times), they were found to diminished. Total IgE levels were determined in 8 of our patients and the median level was 91.1 (minimum 4.47maximum 362) IU/mL. Two patients were observed to have high total IgE values (126 and $362 \mathrm{IU} / \mathrm{mL}$ ) in regard to ageadjusted reference intervals. The laboratory characteristics of patients are presented in Table II. Bone marrow aspiration 


\begin{tabular}{|l|l|l|l|}
\hline \multicolumn{4}{|l|}{ Table II. Laboratory characteristics of patients } \\
\hline $\begin{array}{l}\text { Patient } \\
\text { number }\end{array}$ & $\begin{array}{l}\text { Tryptase } \\
\text { Level (kUA/L) }\end{array}$ & IgE (IU/mL) & $\begin{array}{l}\text { Eosinophil } \\
\text { ratio }\end{array}$ \\
\hline 1 & 4.08 & - & 2.08 \\
\hline 2 & - & 362.00 & 2.3 \\
\hline 3 & 3.6 & 9.96 & 0.33 \\
\hline 4 & 4.3 & 58.90 & 4.2 \\
\hline 5 & 4.84 & - & 1.79 \\
\hline 6 & 8.64 & - & 0.9 \\
\hline 7 & 4.6 & 72.70 & 1 \\
\hline 8 & 7.4 & 126.00 & 4 \\
\hline 9 & 3.6 & 4.47 & 1.4 \\
\hline 10 & 4.41 & - & 7.1 \\
\hline 11 & 16.6 & - & 6.1 \\
\hline 12 & 3.33 & 133.00 & 4.1 \\
\hline 13 & 5.4 & 91.1 & 2.4 \\
\hline
\end{tabular}

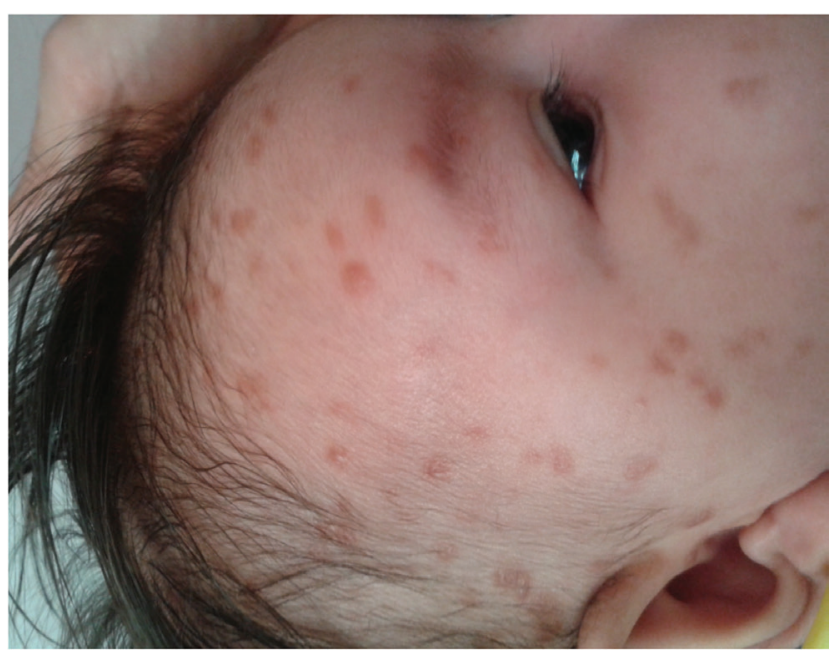

Figure 1. Maculopapuler rash on patient's face

and biopsy were performed on 9 of the 13 patients, none of them indicated an increase of mast cells. All cases were evaluated as cutaneous type mastocytosis. In general, the patients were followed up without any treatment. Oral antihistaminic drugs and local clobetazol proprionate were applied to them during periods of increased pruritis. Patients did not receive any other systemic treatment. In follow-up, there was no increase in the lesions of the patients.

\section{Discussion}

Mastocytosis is a rare disease. While epidemiological studies are insufficient, both in our country and the world, its prevalence is estimated to be 1 in 10,000. Moreover, it has also been reported that approximately 1 in 1,000 to 8,000

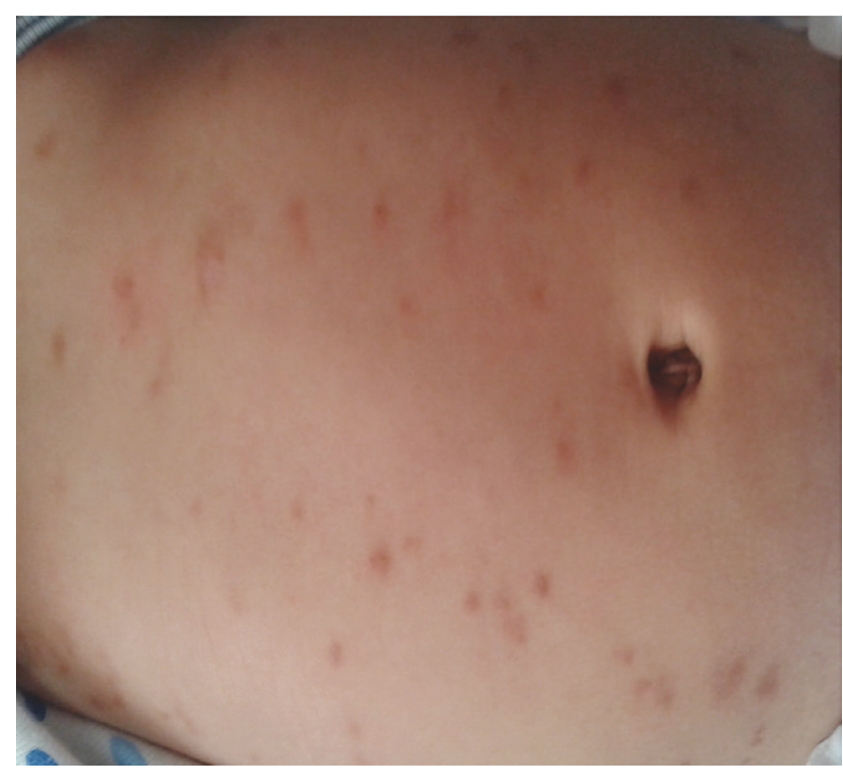

Figure 2. Rash on a patient's trunk

patients who apply to dermatology clinics are diagnosed with mastocytosis (9-11). Mastocytosis can be seen both in childhood and adulthood. In $55 \%$ of cases, the findings and symptoms of the disease appear within the first two years of life, $10 \%$ of patients manifest symptoms between 2-15 years, and the remaining $35 \%$ of the patients show initial findings after 15 years of age. According to a publication from our country, $73 \%$ of mastocytosis cases were reported in children, while $26.7 \%$ were reported in adults $(12,13)$. In our cases, the average age of diagnosis was 20 months and the female/male ratio was $1.1 / 1$. Related literature is conflicting, while some report that mastocytosis is more frequent in females, others indicate that it is more common among males (14-17).

Mastocytosis is now classified with myeloproliferative neoplasms. Increased local concentrations of soluble mast cell growth factor in lesions of CM are believed to stimulate mast cell and melanocyte proliferation. Activating mutations of the proto-oncogene c-kit have been identified. Although pediatric mastocytosis can spontaneously regress, it is a clonal disease most commonly associated with D816V and other activating c-kit mutations. The induction of melanocytes explains the hyperpigmentation that is commonly associated with cutaneous mast cell lesions. The stimulation of pruritus is associated with production of IL-31. IL-6 levels have been shown to be elevated and correlated with the disease severity, indicating IL-6 is involved in the pathogenesis of mastocytosis $(5,6,8)$.

CM is divided to three categories namely maculopapüler form, diffuse CM and solitary mastocytoma (18). CM 
typically manifests as maculopapular or plaque-like rashes seen on the trunk and extremities in the majority of cases, and particularly on the sides of the head and face in children. Figures 1 and 2 show skin rashes in our cases. In both forms of the disease, itching, redness, tachycardia, syncope, gastrointestinal symptoms such as nausea, vomiting, abdominal pain can develop as a result of secretion of mediators such as histamine, heparin and tryptase from the accumulated mast cells. The risk of anaphylaxis is increased in cases with mastocytosis, which is more common in those with SM. In the literature, anaphylactic risk is reported to be $22-50 \%$ in adults and $6-10 \%$ in children. It should be kept in mind that anaphylaxis can often be triggered by fever, infections, and even, albeit rarely, by various foods and medicines during childhood. In its systemic form, symptoms are associated with the affected organs. Hepatomegaly, elevations in liver function tests, acid and portal hypertension may occur due to liver involvement; splenomegaly and hypersplenism may occur in cases with spleen involvement; pancytopenia may develop due to bone marrow involvement; gastrointestinal tract involvement may manifest with hypoalbuminemia and weight loss. More rarely, osteoporosis and pathologic fractures can be seen secondary to skeletal involvement $(1,2,19)$. None of our patients showed any evidence of such findings.

As previously mentioned, SM is defined by major and minor criteria. Major criterion is the multifocal dense infiltrate of mast cells in the bone marrow or in other extracutaneous organ(s). Minor SM criteria are defined as: $25 \%$ of mast cells in bone marrow smears, or are spindleshaped cells in biopsies of extracutaneous organ(s); a KIT mutation at codon 816 in extracutaneous organ(s) or bone marrow; expression of CD2 and/or CD25 in bone marrow or extracutaneous organ(s); or serum tryptase $>20 \mathrm{ng} \mathrm{mL}-1$. We need one major and one minor or at least three minor criteria to diagnose SM $(1,3,8)$. In childhood, it is difficult to determine the exact number of cases with systemic involvement, due to the rareness of the systemic form among children and the fact that bone marrow biopsy is performed in selected cases $(3,4)$. There is no universal consensus on which tests should be performed and how often they should be repeated when mastocytosis is diagnosed in childhood. Contrary to adult mastocytosis, complete blood count, biochemical parameters and peripheral smear findings are normal, as was the case in our study (4). Serum tryptase levels are shown as the most reliable parameter for predicting mast cell activation, and therefore, the severity and prevalence of mastocytosis. Serum tryptase levels are expected to be high especially in patients with very common skin involvement or systemic findings $(20,21)$. Since it is one of the cornerstones of $S M$, without doing bone marrow aspiration and biopsy, diagnosis of some systemic cases may be overlooked. In a large study consisting of 173 patients, the authors found only two cases of systemic involvement in children with diffuse CM (22). In our study, serum tryptase level was at the upper limit of normal in only one patient and follow up measurements were normal. It is known that eosinophils and mast cells coexist in many clonal and nonclonal diseases. Both cells are derived from CD34 + hematopoietic cells and have the ability to regulate their tissue microenvironment. A number of previous studies have shown that eosinophilia can be detected in SM and can indicate an aggressive disease $(23,24)$. Clinical consequence of eosinophilia in SM might largely depend on the subtype of disease and the underlying molecular mechanisms. In one recent study, the total eosinophil ratio was normal. It is also known that IgE levels and IgE-mediated anaphylaxis risk may be increased in patients with mastocytosis $(25,26)$. IgE levels were measured in 8 of our cases, and values were elevated in 2 patients. However, anaphylaxis did not develop in any of our cases.

Our findings show that, even if bone marrow aspiration and biopsy are performed, it is difficult to confirm the diagnosis in patients without conclusive findings for mastocytosis (blood count and smear, increased tryptase, hepatosplenomegaly). Since tryptase activity is an important marker of disease activity, and considering the fact that it was shown to increase beyond the reference range only once in one patient, it may be appropriate to measure tryptase at certain intervals in suspected patients.

\section{Ethics}

Ethics Committee Approval: The study was approved by Acıbadem University Ethics Committee/ATADEK (approvel number:2019-19/11).

Informed Consent: Informed written parental consent was obtained before enrolling children into the study.

Peer-review: Externally and internally peer-reviewed.

\section{Authorship Contributions}

Surgical and Medical Practices: B.A., N.Ö.K., A.Ş.Y., Z.Ö.S., Concept: B.A., Design: B.A., K.K., C.B., D.Y.K., Data Collection or Processing: B.A., H.H.Ö., N.Ö.K., A.Ş.Y., Z.Ö.S., Analysis or Interpretation: B.A., Y.A., Literature Search: B.A., Writing: B.A., D.Y.K.

Conflict of Interest: The authors have no conflicts of interest relevant to this article to disclose. 
Financial Disclosure: The authors have no financial relationships relevant to this article to disclose.

\section{References}

1. Magliacane D, Parente R, Triggiani M. Current concepts on diagnosis and treatment of mastocytosis. Transl Med UniSa 2014;8:65-74.

2. Pardanani A. CME Information: Systemic mastocytosis in adults: 2017 update on diagnosis, risk stratification and management. Am J Hematol 2016;91:1146-59.

3. Valent P, Horny HP, Escribano L, et al. Diagnostic criteria and classification of mastocytosis:a consensus proposal. Leuk Res 2001;25:603-25.

4. Torrelo A, Alwarez-Twose I, Escribano L. Childhood mastocytosis. Curr Opin Pediatr 2012;24:480-6.

5. Kirshenbaum AS, Goff JP, Semere T, Foster B, Scott LM, Metcalfe DD. Demonstration that human mast cells arise from a progenitor cell population that is CD $34(+), c-k i t(+)$, and expresses aminopeptidase N (CD13). Blood 1999;94:2333-42.

6. ValentP, SpanblöchlE, SperrWR, et al. Induction of differentiation of human mast cells from bone marrow and peripheral blood mononuclear cells by recombinant human stem cell factor/kitligand in long-term culture. Blood 1992;80:2237-45.

7. Valent $P$, Akin C, Hartmann K, et al. Advances in the Classification and Treatment of Mastocytosis: Current Status and Outlook toward the Future. Cancer Res 2017;77:1261-70.

8. Valent $P$, Akin $C$, Escribano $M$, et al. Standards and standardization in mastocytosis: Consensus Statements on Diagnostics, Treatment Recommendations and Response Criteria. Eur J Clin Invest 2007;37:435-53.

9. Siebenhaar F, Akin C, Bindslev-Jensen C, Maurer M, BroesbyOlsen S. Treatment Strategies in Mastocytosis. Immunol Allergy Clin North Am 2014;34:433-47.

10. Hartmann K, Henz BM. Mastocytosis: recent advances in defining the disease. Br I Dermatol 2001;144:682-95.

11. Horny HP, Sotlar K, Valent P, Hartmann K. Mastocytosis: a disease of the hematopoietic stem cell. Dtsch Arztebl Int 2008; 105:686-92.

12. Brockow K. Epidemiology, prognosis, and risk factors in mastocytosis. Immunol Allergy Clin North Am 2014;34:283-95.

13. Bilgili SG, Karadağ AS, Takcı Z, Çalka Ö, Kösem M. Comparison of cutaneous mastocytosis with onset in children and adults. Turk J Med Sci 2014:44:504-10.
14. Middelkamp Hup MA, Heide R, Tank B, Mulder PGH, Oranje AP. Comparison of mastocytosis with onset in children and adults. J Eur Acad Dermatol Venereol 2002;16:115-20.

15. Kiszewski AE, Duran-Mckinster C, Orozco-Covarrubias L, Gutierrez-Castrellon P, Ruiz- Maldonado R. Cutaneous mastocytosis in children: a clinical analysis of 71 cases. I Eur Acad Dermatol Venereol 2004;18:285-90.

16. Serarslan G, Atik E, Canda Ş. Pediatric cutaneous mastocytosis: demographic, clinical and histopathological findings. Turk I Dermatol 2008;2:69-72.

17. Ben-Amitai D, Metzker A, Cohen HA. Pediatric cutaneous mastocytosis: a review of 180 patients. Isr Med Assoc I 2005;7:320-22.

18. Hartmann K, Escribano L, Grattan C, et al. Cutaneous manifestations in patients with mastocytosis: Consensus report of the European Competence Network on Mastocytosis; the American Academy of Allergy, Asthma \&lmmunology; and the European Academy of Allergology and Clinical Immunology. I Allergy Clin Immunol 2016;137:35-45.

19. Brockow K, Jofer C, Behrendt H, Ring J. Anaphylaxis in patients with mastocytosis a study on history, clinical features and risk factors in 120 patients. Allergy 2008;63:226-32.

20. Alvarez-Twose I, Vañó-Galván S, Sánchez-Muñoz L, et al. Increased serum baseline tryptase levels and extensive skin involvement are predictors for the severity of mast cell activation episodes in children with mastocytosis. Allergy 2012;67:813-21.

21. Carter MC, Clayton ST, Komarow HD, et al. Assessment of clinical findings, tryptase levels, and bone marrow histopathology in the management of pediatric mastocytosis. I Allergy Clin Immunol 2015;136:1673-9.

22. Hannaford R, Rogers M. Presentation of cutaneous mastocytosis in 173 children. Australas I Dermatol 2001;42:15-21.

23. Kovalszki A, Weller PF. Eosinophilia in mast cell disease. Immunol Allergy Clin N Am 2014;34:357-64.

24. Böhm A, Födinger $M$, Wimazal $F$, et al. Eosinophilia in systemic mastocytosis: clinical and molecular correlates and prognostic significance. I Allergy Clin Immunol 2007;120:192-9.

25. Navinés-Ferrer A, Serrano-Candelas E, Molina-Molina G), Martín M. IgE-related chronic diseases and anti-IgE-based treatments. I Immunol Res 2016;2016:8163803.

26. Escande H, Bennani I, Bulai Livideanu C, Uthurriague C, Paul C, Nougué J. IgE mediated anaphylaxis in a patient with systemic mastocytosis. Ann Dermatol Venereol 2013;140:641-4. 